\title{
ALMOST COMPACTNESS AND DECOMPOSABILITY OF INTEGRAL OPERATORS
}

\author{
WALTER SCHACHERMAYER AND LUTZ WEIS
}

\begin{abstract}
Let $(X, \mu),(Y, \nu)$ be finite measure spaces and $1<q<\infty, 1<p<q$. An integral operator $\operatorname{Int}(k): L^{q}(\nu) \rightarrow L^{P}(\mu)$ becomes compact, if we cut away a suitably chosen subset of $X$ of arbitrarily small measure. As a consequence we prove that $\operatorname{Int}(k)$ may be written as the sum of a Carleman operator and an orderbounded integral operator, where the orderbounded part may be chosen to be compact and of arbitrarily small norm.
\end{abstract}

1. Introduction. $(X, \mathcal{X}, \mu)$ and $(Y, \mathcal{Y}, \nu)$ will denote finite measure spaces. For $1<p, q \leqslant \infty$ we call an operator $T: L^{q}(\nu) \rightarrow L^{p}(\mu)$ integral, if there is a measurable kernel-function $k(x, y)$ on $X \times Y$ such that for $g \in L^{q}(\nu)$

$$
\operatorname{Tg}(x)=\int_{Y} k(x, y) g(y) d \nu(y) \quad \mu \text {-a.e. }
$$

The integrand is required to be Lebesgue-integrable for $\mu$-a.e. $x \in X$ (cf. [7] or [9]). In this case we write $T=\operatorname{Int}(k)$.

There are two well-behaved subclasses of integral operators: $\operatorname{Int}(k)$ is called Carleman if, for $\mu$-a.e. $x \in X, k(x, \cdot) \in L^{r}(\nu)$ where $r^{-1}+q^{-1}=1$. The operator $\operatorname{Int}(k)$ is called orderbounded if it transforms orderbounded sets into orderbounded sets or equivalently if $|k|$ also defines an integral operator from $L^{q}(\nu)$ to $L^{p}(\mu)$. In this case we call $\operatorname{Int}(|k|)$ the modulus or absolute value of $\operatorname{Int}(k)$.

Let us specify the following notation. If $g \in L^{\infty}(\mu)$ we denote by $P_{g}$ the multiplication operator $f \rightarrow f \cdot g$ on $L^{P}(\mu)$. If $g=\chi_{A}$ is a characteristic function we write $P_{A}$ for $P_{\chi_{1}}$.

2. Preliminaries. In this section we recall known results for later reference.

2.1. Theorem (NIKIŠIn, [11, Theorem 4]). Let $0<q<\infty$ and $T: L^{q}(\nu) \rightarrow L^{0}(\mu)$ be a positive, continuous operator. For $\varepsilon>0$ there is an $A \subseteq X, \mu(X \backslash A)<\varepsilon$ and such that $P_{A} \circ T$ takes its values in $L^{q}(\mu)$.

2.2. Theorem (Maurey, [10, Proposition 9]). Let $0<p<q<\infty$ and $T: L^{q}(v)$ $\rightarrow L^{p}(\mu)$ be a positive, continuous operator. For $r^{-1}=p^{-1}-q^{-1}$ there is a strictly positive function $g \in L^{\infty}(\mu)$ such that $g^{-1} \in L^{r}(\mu)$ and $P_{g} \circ T$ takes its values in $L^{q}(\mu)$.

We also need a technical result, which follows easily from [9, Theorems 4.7 and 5.12].

Received by the editors January $15,1980$.

1980 Mathematics Subject Classification. Primary 47G05; Secondary 47B10.

Key words and phrases. Integral operator 
2.3. Lemma. Let $1<q<\infty, 1<p<\infty$ and $k(x, y)>0$ be such that $\operatorname{Int}(k)$ defines an operator from $L^{q}(\nu)$ to $L^{p}(\mu)$. Let $k_{n}(x, y)>0$ be such that $k=\sum_{n=1}^{\infty} k_{n}$.

(a) $\sum_{n=1}^{\infty} \operatorname{Int}\left(k_{n}\right)$ converges unconditionally to $\operatorname{Int}(k)$ in the strong operator topology of $B\left(L^{q}(\nu), L^{p}(\mu)\right)$.

(b) If $1<q<\infty$ and $\operatorname{Int}(k)$ is compact then the above sum converges unconditionally in the norm of $B\left(L^{q}(\nu), L^{p}(\mu)\right)$.

\section{Almost compactness of positive integral operators.}

3.1. TheOREM. Let $1<q<\infty$ and $k(x, y)>0$ be such that $\operatorname{Int}(k)$ defines an operator from $L^{q}(\nu)$ to $L^{q}(\mu)$. Given $r<\infty$ we may find $g \in L^{\infty}(\mu)$ such that $g^{-1} \in L^{r}(\mu)$ and $P_{g} \circ \operatorname{Int}(k): L^{q}(\nu) \rightarrow L^{q}(\mu)$ is compact.

Proof. Let us start with the easy case $q=\infty$. It is an old result, dating back to Dunford's paper [4] in 1936, that a $\sigma^{*}$-continuous $T: L^{\infty}(\nu) \rightarrow L^{\infty}(\mu)$ is integral iff for $\varepsilon>0$ there is $A \subseteq X, \mu(X \backslash A)<\varepsilon$ and such that $P_{A} \circ T$ is compact (see also [5] and [12]). So find a partition $\left(A_{n}\right)_{n=1}^{\infty}$ of $X$ such that $P_{A_{n}} \circ \operatorname{Int}(k)$ is compact and, given $r<\infty$, find a nullsequence $\left(\alpha_{n}\right)_{n=1}^{\infty}$ of strictly positive scalars such that $g^{-1}=\sum_{n=1}^{\infty} \alpha_{n}^{-1} \chi_{A_{n}} \in L^{r}(\mu)$. It is easy to check that $P_{g} \circ \operatorname{Int}(k)$ is compact.

Now assume that $1<q<\infty$. Given $r<\infty$ find $1<p<q$ such that $r^{-1}>p^{-1}$ $-q^{-1}$. The operator $\operatorname{Int}(k)$ is a compact operator from $L^{q}(\nu)$ to $L^{p}(\mu)$ (cf. [1] or [9, Theorem 5.4]; compare also [3]). Let $k_{n}=k \cdot \chi_{\{n-1<k<n\}}$ and deduce from 2.3(b) that $\sum_{n=1}^{\infty} \operatorname{Int}\left(k_{n}\right)$ converges to $\operatorname{Int}(k)$ unconditionally in the norm of $B\left(L^{q}(\nu), L^{p}(\mu)\right)$. So we may find a sequence $0=n_{0}<n_{1}<\cdots<n_{m}<\ldots$ such that for $m>2$

$$
\left\|\sum_{n=n_{m-1}}^{n_{m}-1} \operatorname{Int}\left(k_{n}\right)\right\|_{B\left(L^{q}, L^{p}\right)}<2^{-m} .
$$

Let $\bar{k}_{m}=m \sum_{n=n_{m-1}}^{n_{m}-1} k_{n}$, and $\bar{k}=\sum_{m=1}^{\infty} \bar{k}_{m}$. Clearly $\bar{k} \geqslant k$ but $\operatorname{Int}(\bar{k})=\sum_{m=1}^{\infty} \operatorname{Int}\left(\bar{k}_{m}\right)$ is still a continuous (even compact, but we shall not need this) operator from $L^{q}(\nu)$ to $L^{p}(\mu)$. We may apply Maurey's factorization theorem (2.2 above) to find $g \in L^{\infty}(\mu)$ such that $g^{-1} \in L^{r}(\mu)$ and such that $P_{g} \circ \operatorname{Int}(\bar{k})$ takes its values in $L^{q}(\nu)$. From 2.3(a)

$$
P_{g} \circ \operatorname{Int}(\bar{k})=\sum_{m=1}^{\infty} m\left(\sum_{n=n_{m-1}}^{n_{m}-1} \operatorname{Int}\left(g \cdot k_{n}\right)\right),
$$

the sum converging unconditionally in the strong operator topology of $B\left(L^{q}(\nu), L^{q}(\mu)\right)$. This implies that the sum

$$
P_{g} \circ \operatorname{Int}(k)=\sum_{m=1}^{\infty}\left(\sum_{n=n_{m-1}}^{n_{m}-1} \operatorname{Int}\left(g \cdot k_{n}\right)\right)
$$

converges in the norm of $B\left(L^{q}(\nu), L^{q}(\mu)\right)$. As each of the summands is clearly compact the operator $P_{g} \circ \operatorname{Int}(k): L^{q}(\nu) \rightarrow L^{q}(\mu)$ is compact.

3.2. Remark. The theorem does not hold for $q=1$. Let $T: L^{1}(\nu) \rightarrow L^{1}[0,1]$ be a positive surjective operator, where $(Y, \nu)$ is a purely atomic measure space (i.e. 
$L^{1}(\nu)$ is isometric to $\left.l^{1}\right)$. Then $T$ is integral but for every positive $g \in L^{\infty}(\mu)$, which does not vanish identically, the operator $P_{g} \circ \operatorname{Int}(k)$ is not compact.

However, we have the following result by duality.

3.3. Corollary. Let $1<p<\infty$ and $k(y, x)>0$ such that $\operatorname{Int}(k)$ defines an operator from $L^{p}(\mu)$ to $L^{p}(\nu)$. Given $r<\infty$ we may find $g \in L^{\infty}(\mu)$ such that $g^{-1} \in L^{r}(\mu)$ and $\operatorname{Int}(k) \circ P_{g}: L^{p}(\mu) \rightarrow L^{p}(\nu)$ is compact.

\section{Almost compactness of general integral operators.}

4.1. Theorem. Let $1<q<\infty$ and $1<p<q$ and let $\operatorname{Int}(k): L^{q}(\nu) \rightarrow L^{p}(\mu)$ be an integral operator. For $\varepsilon>0$ there is $A \subseteq X$ with $\mu(X \backslash A)<\varepsilon$ such that both $P_{A} \circ \operatorname{Int}(k)$ and its modulus $P_{A} \circ \operatorname{Int}(|k|)$ are compact operators from $L^{q}(\nu)$ to $L^{q}(\mu)$.

Proof. Write $k=k_{1}-k_{2}+i k_{3}-i k_{4}$, where $k_{j}>0$. Each $\operatorname{Int}\left(k_{j}\right)$ defines a positive continuous operator from $L^{q}(\nu)$ to $L^{0}(\mu)$. By Nikišin's theorem (2.1 above) we may find $B_{j} \subseteq X, \mu\left(X \backslash B_{j}\right)<\varepsilon / 8$ such that $P_{B_{j}} \circ \operatorname{Int}\left(k_{j}\right)$ is a positive continuous operator from $L^{q}(\nu)$ to $L^{q}(\mu)$. It is an easy consequence of Theorem 3.1 that we may find $A_{j} \subseteq B_{j}, \mu\left(X \backslash A_{j}\right)<\varepsilon / 4$, such that $P_{A} \circ \operatorname{Int}\left(k_{j}\right)$ is compact from $L^{q}(\nu)$ to $L^{q}(\mu)$. For $A=\cap{ }_{j=1}^{4} A_{j}$, the operator $P_{A} \circ \operatorname{Int}(k)$ satisfies the requirements.

4.2. Remark. In the situation of Theorem 4.1, it is not possible to find a big set $B$ on the left-hand side (i.e. from $Y$ ) so that $\operatorname{Int}(k) \circ P_{B}$ is compact. For example let $k$ be the kernel on $[0,1] \times[0,1], k(x, y)=2^{n / 2} \cdot r_{n}(y)$ if $x \in\left[2^{-n}, 2^{-(n-1)}\right]$, where $r_{n}$ denotes the $n$th Rademacher function. Then $\operatorname{Int}(k): L^{2}[0,1] \rightarrow L^{2}[0,1]$ is such an example.

Theorem 4.1 is a strengthening of the known result of "twosided cutting off", which seems to be due to Korotkov [8].

4.3. REMARK. What happens in the case $p>q$ ? If $\operatorname{Int}(k): L^{q}(\nu) \rightarrow L^{p}(\mu)$ is given, then for $q>1$ the above theorem applies and provides a compact operator $P_{A} \circ \operatorname{Int}(k)$ from $L^{q}(\nu)$ to $L^{q}(\mu)$. One would like to have the operator compact from $L^{q}(\nu)$ to $L^{p}(\mu)$ but this is only possible for few pairs of indices as is shown in the following proposition.

4.4. Proposition. (a) Let $1<q<\infty$ and $p=\infty$; for every continuous operator T: $L^{q}(\nu) \rightarrow L^{\infty}(\mu)$ and $\varepsilon>0$ there is an $A \subseteq X$ with $\mu(X \backslash A)<\varepsilon$ such that $P_{A} \circ T: L^{q}(\nu) \rightarrow L^{\infty}(\mu)$ is compact.

(b) On the other hand, for $1 \leqslant q<p<\infty$ and for $q=1, p=\infty$ there are integral operators $\operatorname{Int}(k): L^{q}(\nu) \rightarrow L^{p}(\mu)$ such that for every $A \subseteq X, \mu(A)>0$ the operator $P_{A} \circ T: L^{q}(\nu) \rightarrow L^{p}(\mu)$ is not compact.

Proof. (a) This result was known to A. Grothendieck [6]. Let us phrase it in the terminology of [13]: $L^{q}(\nu)$ is Asplund for $1<q<\infty$ hence $T$ (ball $\left(L^{q}(\nu)\right)$ ) is equimeasurable, which is just what we have to prove.

(b) For $q=1$ and $1<p<\infty$ let $T$ be a positive surjective operator from $l^{1}$ (represented as $L^{1}(\nu)$ over a finite measure space $(Y, \nu)$ ) onto $L^{p}[0,1]$ (resp. onto the subspace $C[0,1]$ of $L^{\infty}[0,1]$, if $\left.p=\infty\right)$. 
If $1<q<p<\infty$ then there are operators of potential type from $L^{q}[0,1]$ to $L^{p}[0,1]$ that are not compact (cf. [8, p. $147 \mathrm{ff}$.]). It is clear that an operator of potential type may not be made compact by restricting to a subset of positive measure.

\section{Decomposition of integral operators.}

5.1. TheOREM. Let $1<q \leqslant \infty, 1 \leqslant p<q$ and $\operatorname{Int}(k): L^{q}(\nu) \rightarrow L^{p}(\mu)$ an integral operator. Given $\varepsilon>0$ we may write $k$ as $k^{C}+k^{0}$ where $\operatorname{Int}\left(k^{C}\right)$ is a Carleman operator from $L^{q}(\nu)$ to $L^{p}(\mu)$ and $\operatorname{Int}\left(k^{0}\right)$ as well as its modulus $\operatorname{Int}\left(\left|k^{0}\right|\right)$ are compact operators from $L^{q}(\nu)$ to $L^{q}(\mu)$ of norm less than $\varepsilon$.

Proof. We start with the trivial case $q=\infty$ and $1<p<\infty$. Every $\operatorname{Int}(k)$ : $L^{\infty}(\nu) \rightarrow L^{p}(\mu)$ is automatically Carleman, hence we may choose $k^{C}=k$ and $k^{0}=0$.

Let now $1<q<\infty, 1 \leqslant p \leqslant q$. By Theorem 4.1 we may find a partition $\left(A_{i}\right)_{i=1}^{\infty}$ of $X$ such that for $k_{i}(x, y)=\chi_{A_{i}}(x) \cdot k(x, y)$ the operator $\operatorname{Int}\left(\left|k_{i}\right|\right)$ is compact from $L^{q}(\nu)$ to $L^{q}(\mu)$. By Lemma 2.3 we may find numbers $n_{i}$ such that

$$
\left\|\operatorname{Int}\left(\left|k_{i}\right|\right)-\operatorname{Int}\left(\left|k_{i}\right| \cdot \chi_{\left\{\left|k_{i}\right|<n_{i}\right\}}\right)\right\|<\varepsilon / 2^{i} .
$$

Let $k_{i}^{C}=k_{i} \cdot \chi_{\left\{\left|k_{i}\right|<n_{i}\right\}}$ and $k_{i}^{0}=k_{i}-k_{i}^{C}$ and define

$$
k^{c}=\sum_{i=1}^{\infty} k_{i}^{c} \text { and } k^{0}=\sum_{i=1}^{\infty} k_{i}^{0} .
$$

It is now easy to verify the asserted properties of $k^{C}$ and $k^{0}$.

5.2. Remark. We do not know whether for arbitrary $1<p, q<\infty$ an integral operator $\operatorname{Int}(k): L^{q}(\nu) \rightarrow L^{p}(\mu)$ may be decomposed into a Carleman and an orderbounded part. We know that this is possible in some cases not covered by 5.1 . For $p=q=1$, for example, this is trivially possible as every continuous operator $T: L^{1}(\nu) \rightarrow L^{1}(\mu)$ is orderbounded. However, we do not have the full strength of 5.1 in this case. The operator from Remark 3.2 may not be decomposed in such a way as to make the orderbounded part compact or arbitrarily small in norm.

\section{REFERENCES}

1. T. Ando, On compactness of integral operators, Indag. Math. 24 (1962), 235-239.

2. J. Diestel and J. J. Uhl, Vector measures, Math. Surveys, no. 15, Amer. Math. Soc., Providence, R.I., 1977.

3. P. G. Dodds, Compact kernel operators on Banach function spaces, (preprint).

4. N. Dunford, Integration and linear operations, Trans. Amer. Math. Soc. 40 (1936), 474-494.

5. N. E. Gretsky and J. J. Uhl, Carleman and Korotkov operators on Banach spaces, (preprint).

6. A. Grothendieck, Produits tensoriels topologiques, Mem. Amer. Math. Soc. No. 16 (1955).

7. P. R. Halmos and V. Sunder, Bounded integral operators on $L^{2}$ spaces, Ergebnisse der Math., Bd. 96, Springer-Verlag, Berlin and New York, 1978.

8. V. Korotkov, On some properties of partially integral operators, Dokl. Akad. Nauk SSSR 217 (1974); English transl. in Soviet Math. Dokl. 15 (1974), 1114-1117.

9. M. A. Krasnosel'skii et al., Integral operators in spaces of summable functions, Nordhoff, Groningen, 1976. 
10. B. Maurey, Théorèmes de factorisation pour les operateurs linéaires à valeurs dans $L^{p}$, Asterisque 11 , Paris, 1974.

11. E. M. Nikišin, Resonance theorems and superlinear operators, Úspehi Mat. Nauk 25 (1970), 125-191; English transl. in Russian Math. Surveys 25 (1970), 125-197.

12. W. Schachermayer, Integral operators on $L^{p}$ spaces, (preprint).

13. C. Stegall, The Radon-Nikodym property in conjugate Banach spaces. II, Trans. Amer. Math. Soc. (to appear)

INSTITUT Für MATHEMATIK, JOHANNES KePLER UNIVERSITÄT, A 4045 LINZ, AUSTRIA

Fachbereich Mathematix, Universttät Kaiserslautern, 675 Kaiserslautern, Federal RepubLIC OF GERMANY 